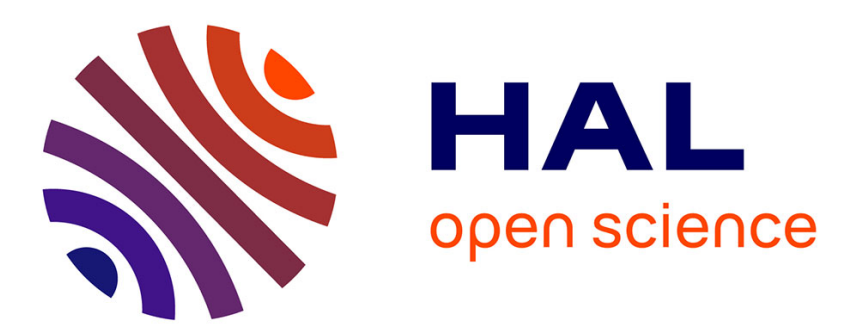

\title{
Intramolecular amidoalkylation cyclizations in synthesis of novel pyrrolo(or isoindolo)thieno[2] benzazepines
}

Aïcha Mamouni, Pascal Pigeon, Abdel Adam Daïch, Bernard Decroix

\section{To cite this version:}

Aïcha Mamouni, Pascal Pigeon, Abdel Adam Daïch, Bernard Decroix. Intramolecular amidoalkylation cyclizations in synthesis of novel pyrrolo(or isoindolo)thieno[2]benzazepines. Journal of Heterocyclic Chemistry, 1997, 34 (5), pp.1495-1499. 10.1002/jhet.5570340519 . hal-01230424

\section{HAL Id: hal-01230424 \\ https://hal.science/hal-01230424}

Submitted on 19 Oct 2017

HAL is a multi-disciplinary open access archive for the deposit and dissemination of scientific research documents, whether they are published or not. The documents may come from teaching and research institutions in France or abroad, or from public or private research centers.
L'archive ouverte pluridisciplinaire HAL, est destinée au dépôt et à la diffusion de documents scientifiques de niveau recherche, publiés ou non, émanant des établissements d'enseignement et de recherche français ou étrangers, des laboratoires publics ou privés. 


\title{
Intramolecular Amidoalkylation Cyclizations in Synthesis of Novel Pyrrolo(or Isoindolo)thieno[2]benzazepines
}

\author{
Aicha Mamouni, Pascal Pigeon, Adam Daïch and Bernard Decroix* \\ Laboratoire de Chimie, Faculté des Sciences et Techniques de l'Université du Havre, 30 rue \\ Gabriel Péri, 76600 Le Havre, France \\ Thieno[2] benzazepines annulated to pyrrole $\mathbf{8 a}, \mathbf{b}$ or isoindole $\mathbf{8 c}$ have been synthesized \\ utilizing the acid-catalyzed cyclization of hydroxylactams $\mathbf{7 a - c}$ as key step.
}

As a part of our program aimed at the synthesis of products which have most potent CNS activity, we have previously reported some thieno[2]azepinones annulated to isoindole [1] or pyrrolidine [2] rings. These compounds are structurally related to some natural isoindolo(or pyrrolo)benzazepine alkaloids: and derivatives [3] isolated from Chilean Berberidaceae, Berberis darwinii Hook and Taxus baccata [4]. On the other hand, it was found that prominent examples of structures which bear a benzazepine moiety such as pyridobenzazepinone [5], hexahydrodibenzo[c,f]pyrazino[1,2- $d$ ] azepine [6] and trans-2,3,5,6,11,11a-hexahydro-6phenyl-1 $H$-pyrrolo[2,1-b][3]benzazepine [7], exhibit more significant clinical antidepressant effects. Furthermore, any number derivatives of dibenzo[c,e; $b, e ; b, f]$ azepines are known and display hypolipidermic activity [8], CNS activity [9], and antiarrhythmic activity [10], respectively. In view of our interest in the synthesis of diversely substituted polycyclic systems, we wish to report herein an interesting approach to thieno[2]benzazepines annulated to a pyrrolidinone moiety as $\mathbf{8 a , b}$ or annulated to an isoindole moiety as $\mathbf{8 c}$.

We recently developed a method for the preparation of indolizidines annulated to both thiophene and benzene rings. The Heck reaction of aryl radical cyclization of suitable enamides was the key step [11]. From this result and in a similar manner we thought to arrive to the thieno[2]benzazepines 8a-c. Unfortunately, whereas the addition of a Grignard reagent (phenylmagnesium bromide) to the imide function of the reported phthalimide derivative $\mathbf{3 c}$ [1] gave the expected alcohol 4c (65\%) (Scheme 1), no reaction was observed with the imides $\mathbf{3 a}, \mathbf{b}$. The resulting hydroxylactam $\mathbf{4 c}$ was reduced using triethylsilane in trifluoroacetic acid to afford lactam $\mathbf{5 c}$ in a good yield of $91 \%$ (Scheme 1).

As shown in Scheme 1, the lactam 5c was subjected to the usual radical cyclization conditions (2,2'-azobisisobutyronitrile, tributyltin hydride, toluene) without success. Likewise, an intramolecular Heck reaction did not give the expected 
isoindolothieno[2]benzazepine 8c. These failures were similar to those reported in the literature for related compounds $[12,13,14]$. Nevertheless a partial success had been observed for cyclization, under the action of tri- $n$-butyltin hydride, of a non-conjugate enamide to a 7 membered ring [15].

Scheme 1<smiles>O=C1c2ccccc2C(=O)N1Cc1ccsc1Br</smiles>

$3 c$<smiles>O=C1c2ccccc2C2c3ccccc3-c3sccc3CN12</smiles>

$8 \mathrm{c}$<smiles>O=C1c2ccccc2C(O)(c2ccccc2)N1Cc1ccsc1Br</smiles>

$4 \mathrm{c}$

$\mathrm{El}_{3} \mathrm{SiH} / \mathrm{TFA}$

$\mathrm{Pd}\left(\mathrm{PPh}_{3}\right)_{4} / \mathrm{CH}_{3} \mathrm{CN}$

or $\mathrm{Bu}_{3} \mathrm{SnH} / \mathrm{AIBN}$<smiles>O=C1c2ccccc2C(c2ccccc2)N1Cc1ccsc1Br</smiles>

$5 c$

From these results we were then led to examine another approach using $N$-acyliminiumaromatic cyclization. Actually, heterocyclization involving $\mathrm{N}$-acyliminium ions (intramolecular $\alpha$-amidoalkylation reaction) has been widely studied [16]. In this field, we recently presented our preliminary studies concerning the synthesis of dibenzo[c,e]azepines [17]. Our results were in accordance with the Baldwin's rules [18]. Since a 7-endo-trigonal ring forming process is favored rather than a 5-endo-trigonal one, the hydroxylactams 7a-c (Scheme 2) should lead to the thienobenzazepines 8a-c. $N$-acyliminium ion precursors 7a-c were obtained from phthalimide derivative $\mathbf{3 c}$ or succinimide derivatives $\mathbf{3 a}, \mathbf{b}$. The latter compounds were prepared by alkylation of the commercially available succinimide (2a) and tetramethylsuccinimide (2b) [19] with 2-bromo-3-bromomethylthiophene (1) [20] under 
solid-liquid phase transfer catalysis using anhydrous potassium carbonate as base and a mixture of potassium iodide and 18-crown-6 as the catalysts [21].

Scheme 2

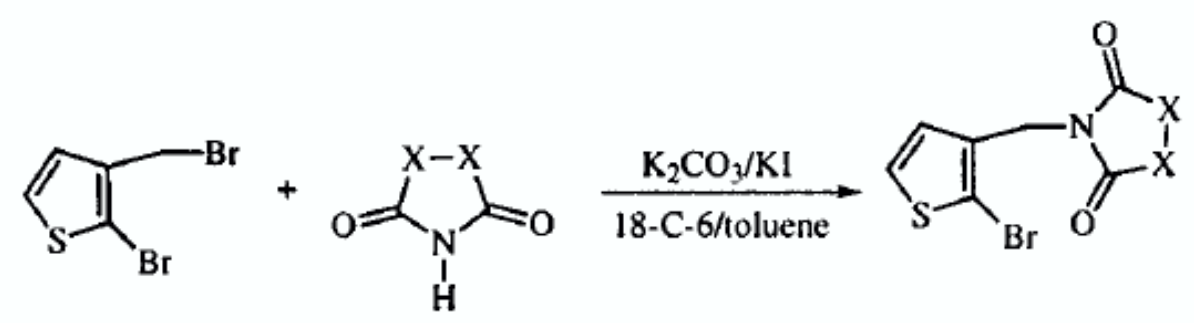

1

2a,b

3a,b,c

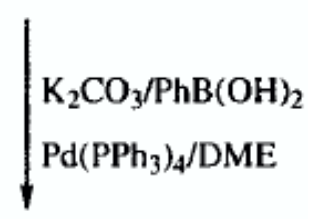<smiles>[X]C1CC(O)N(Cc2ccsc2-c2ccccc2)C1=O</smiles>

7a,b,c<smiles>[X]C1=C([Y])N2Cc3ccsc3-c3ccccc3C1[Y]2=O</smiles>

$\mathrm{NaBH}_{4} / \mathrm{MeOH}$

6a,6c: $\mathrm{HCl} / \mathrm{E} \mathrm{OH} ;-5^{\circ}$ to $0^{\circ}$

6b: $\mathrm{HCl} / \mathrm{EtOH} ; \mathrm{rt}$<smiles></smiles>

6a,b,c

\section{$\mathrm{X}-\mathrm{X} \quad \mathrm{CH}_{2}-\mathrm{CH}_{2} \quad \mathrm{C}(\mathrm{Me})_{2}-\mathrm{C}(\mathrm{Me})_{2}$}<smiles>c1ccccc1</smiles>

Product

a

b

c

8a,b,c

A palladium(0) catalyzed cross-coupling of bromo derivatives 3a-c with phenylboronic acid provided the diaryl compounds 6a-c in high yields (95 to 98\%). Reduction of imides 6a-c by sodium borohydride in anhydrous methanol and in the presence of acid, to avoid the formation of the opened amide-alcohol [2,22], afforded the hydroxylactams 7a-c (85 to 96\%). Speckamp reported $[22,23]$ that with succinimides bearing two relatively large substituents at a single carbon atom, the reduction did not need addition of acid. Likewise in the case of 
tetramethylsuccinimide derivative $\mathbf{6 b}$, in the absence of hydrochloric acid we have never observed the ring opened amide-alcohol.

Hydroxylactams 7a-c were then subjected to trifluoroacetic acid in dry dichloromethane at room temperature [11], and led exclusively to the thienobenzazepines 8a-c. This reaction provides a new example of $\mathrm{N}$-acyliminium cyclization resulting in a nitrogen containing 7membered ring. The structures of 8a-c were assigned on the basis of their mass, ir, and nmr $\left({ }^{1} \mathrm{H}\right.$ and $\left.{ }^{13} \mathrm{C}\right)$ spectra as well as by their microanalyses. Thus, the two protons of the thiophene ring appear as doublets ( $\mathrm{AB}$ system) with a coupling constant of $5 \mathrm{~Hz}$ characteristic of $\alpha, \beta-$ substituted thiophene. Furthermore in the ${ }^{1} \mathrm{H}$ nmr spectra of $\mathbf{8 a - c}$ the methylene protons $\left(\mathrm{CH}_{2}\right.$ $\mathrm{N}$ ) appear as an $\mathrm{AB}$ system due to the diastereotopic effect with a coupling constant of about $15 \mathrm{~Hz}$ characteristic of gem protons. Likewise, the key feature in the ${ }^{13} \mathrm{C} \mathrm{nmr}$ spectra of 8a-c was the appearance of ten signals for $\mathbf{8 a}$ and $\mathbf{8 b}$ and sixteen signals for $\mathbf{8 c}$ in the aromatic region. Moreover, one of these resonances disappears in the corresponding DEPT program spectra, as a consequence of the intramolecular amidoalkylation cyclization.

The present work shows a general methodology for the synthesis of biaryl $[c, e]$ azepines annulated to an isoindole or pyrrolidinone moiety starting from hydroxylactams and according to the Baldwin's rules the cyclization process gives a 7-membered ring rather than a 5-membered ring.

EXPERIMENTAL

All melting points were determined using a Leitz heat plate apparatus and are uncorrected. Infrared spectra were recorded on a Perkin Elmer FT-IR paragon 1000 spectrometer. The nuclear magnetic resonance spectra $\left({ }^{1} \mathrm{H}\right.$ and $\left.{ }^{13} \mathrm{C}\right)$ were taken on a Bruker AC-200 $(200 \mathrm{MHz})$ instrument in the solvent indicated. Chemical shifts values are reported in ppm from TMS as an internal reference and are given in $\delta$ units and the following abbreviations are used: $\mathrm{s}$ for singlet, $\mathrm{d}$ for doublet, dd for doublet of doublet, br for broad and finally $\mathrm{m}$ for multiplet. Elemental analyses were obtained in the microanalysis laboratory of the I.N.S.A at Rouen, F 76130 Mt-St-Aignan. Mass spectral measurements were recorded on an AEI MS 902 S Spectrometer. Thin layer chromatography was performed on precoated plates of silica gel 60F 254 (Merck) and the spots visualized using an ultraviolet lamp or iodine vapor. E. Merck silica gel 
60F (70-300 mesh) was used for column chromatography. The starting materials $\mathbf{1}, \mathbf{2 b}$ and $\mathbf{3 c}$ were prepared according to known procedures $[1,19,20]$.

General Procedure for $N$-alkylation of Halides 2a,b.

To a mixture of succinimide (2a) or tetramethylsuccinimide (2b) (10 mmoles) and 18crown-6 $(1 \% \mathrm{w} / \mathrm{w})$ in dry toluene $(15 \mathrm{ml})$ was added solid potassium carbonate $(1.34 \mathrm{~g}, 11$ mmoles) and 0.1 equivalent per mmole of potassium iodide. After stirring for 10 minutes, 2bromo-3-bromomethylthiophene (1) (3.07 g, $12 \mathrm{mmoles})$ in dry toluene (10 ml) was added slowly dropwise over a period of 15 minutes. The mixture was then refluxed for 24 hours under nitrogen atmosphere and cooled. The heterogeneous solution was filtered over a short column of Celite which was washed with toluene $(3 \times 10 \mathrm{ml})$. The organic phase was evaporated in vacuo and the resulting crude solid was recrystallized from anhydrous ethanol.

1-(2'-Bromothien-3'-ylmethyl)succinimide (3a).

This product was isolated as colorless needles in $92 \%$ yield, mp $110^{\circ} ; \mathrm{R}_{\mathrm{f}} 0.87$ (dichloromethane/acetone, 9:1); ir: $\quad$ v $2896(\mathrm{CH}), \quad 1653 \quad(\mathrm{C}=\mathrm{O}) \quad \mathrm{cm}^{-1} ; \quad{ }^{1} \mathrm{H} \quad \mathrm{nmr}$ (deuteriochloroform): $\delta 2.70$ (s, 4H, 4H-succinimide), $4.62(\mathrm{~s}, 2 \mathrm{H}, \mathrm{CH}-\mathrm{N}), 6.90(\mathrm{~d}, 1 \mathrm{H}, \mathrm{J}=$ $5.6 \mathrm{~Hz}, \mathrm{H}_{4}$-thiophene), $7.16\left(\mathrm{~d}, \quad 1 \mathrm{H}, \quad \mathrm{J}=5.6 \mathrm{~Hz}, \quad \mathrm{H}_{5}\right.$,-thiophene $) ;{ }^{13} \mathrm{C} \mathrm{nmr}$ (deuteriochloroform): $\delta 28.0\left(2 \mathrm{CH}_{2}\right), 36.4\left(\mathrm{CH}_{2}\right), 111.7(\mathrm{C}), 125.9(\mathrm{CH}), 128.3(\mathrm{CH}), 134.9$ (C), 176.3 (2CO); ms: (EI) m/z $274\left(\mathrm{M}^{+}\right)$.

Anal. Calcd. for $\mathrm{C}_{9} \mathrm{H}_{8} \mathrm{BrNO}_{2} \mathrm{~S}$ (274.13): C, 39.43; H, 2.94; N, 5.11. Found: C, 39.29; H, $2.89 ; \mathrm{N}, 4.99$.

3,3,4,4-Tetramethyl-1-(2'-bromothien-3'-ylmethyl)succinimide (3b).

This product was obtained similarly from $\mathbf{1}$ and $\mathbf{2 b}$ in a yield of $85 \%, \mathrm{mp} 66^{\circ} ; \mathrm{R}_{\mathrm{f}} 0.45$ (chloroform/acetone, 9:1); ir: $v 2942(\mathrm{CH}), 1677(\mathrm{C}=\mathrm{O}) \mathrm{cm}^{-1} ;{ }^{1} \mathrm{H} \mathrm{nmr}$ (deuteriochloroform): $\delta 1.10\left(\mathrm{~s}, 12 \mathrm{H}, 4 \mathrm{CH}_{3}\right), 4.60\left(\mathrm{~s}, 2 \mathrm{H}, \mathrm{CH}_{2}-\mathrm{N}\right), 7.64$ (d, $1 \mathrm{H}, \mathrm{J}=5.6 \mathrm{~Hz}, \mathrm{H}_{4}$,-thiophene), 7.29 (d, $1 \mathrm{H}, \mathrm{J}=5.6 \mathrm{~Hz}, \mathrm{H}_{5}$,-thiophene); ${ }^{13} \mathrm{C} \mathrm{nmr}$ (deuteriochloroform): $\delta 21.2\left(4 \mathrm{CH}_{3}\right), 36.3\left(\mathrm{CH}_{2}\right)$, $46.8(2 \mathrm{C}), 116.6(\mathrm{C}), 126.1(\mathrm{CH}), 127.6(\mathrm{CH}), 135.2(\mathrm{C}), 181.1(2 \mathrm{CO}) ; \mathrm{ms}:(\mathrm{EI}) \mathrm{m} / \mathrm{z} 330$ $\left(\mathrm{M}^{+}\right)$.

Anal. Calcd. for $\mathrm{C}_{13} \mathrm{H}_{16} \mathrm{BrNO}_{2} \mathrm{~S}$ (330.24): C, 47.28; H, 4.88; N, 4.24. Found: C, 47.12; H, $4.69 ; \mathrm{N}, 4.15$.

2,3-Dihydro-3-hydroxy-3-phenyl-2-(2'-bromothien-3'-ylmethyl)-1 $H$-isoindol-1-one (4c).

To a stirred solution of (2'-bromothien-3'-ylmethyl)-l-phthalimide (3c) (3.23 g, 10 mmoles) in $100 \mathrm{ml}$ of dry dichloromethane was added dropwise over a period of 10 minutes $3.63 \mathrm{~g}$ (20 
mmoles) of phenylmagnesium bromide (prepared by classical procedure from magnesium (0.535 g, 22 g-atoms) and phenyl bromide (3.14 g, 20 mmoles) in dry tetrahydrofuran). After 3 hours of reaction at room temperature, the reaction mixture was poured into a solution of $20 \%$ ammonium chloride $(100 \mathrm{ml})$ and decanted. The organic phase was washed with water, brine, dried over magnesium sulfate and concentrated in vacuo. The resulting solid was purified by chromatography on silica gel column eluting with dichloromethane/hexane $(9: 1)$ ( $R f(0.78)$ to isolate $\omega$-carbinol lactam $4 \mathbf{c}$ as white crystals in a yield of $65 \%, \mathrm{mp} 182^{\circ}$; ir: $v$ 3270 (br, O-H), $1667(\mathrm{C}=\mathrm{O}) \mathrm{cm}^{-1}$; ${ }^{1} \mathrm{H}$ nmr (deuteriochloroform): $\delta 3.30$ (s, 1H, O-H), 4.16 (d, $\left.1 \mathrm{H}, \mathrm{J}=15.3 \mathrm{~Hz}, \mathrm{CH}_{2}-\mathrm{N}\right), 4.57\left(\mathrm{~d}, 1 \mathrm{H}, \mathrm{J}=15.3 \mathrm{~Hz}, \mathrm{CH}_{2}-\mathrm{N}\right), 6.88\left(\mathrm{~d}, 1 \mathrm{H}, \mathrm{J}=5.6 \mathrm{~Hz}, \mathrm{H}_{4^{-}}\right.$ thiophene), 7.03 (d, 1H, J = 5.6 Hz, $\mathrm{H}_{5}$-thiophene), 7.17-7.32 (m, 6H, 6H-benzene), 7.41-7.49 (m, 2H, 2H-benzene), 7.74-7.82 (m, 1H, 1H-benzene); ${ }^{13} \mathrm{C} \mathrm{nmr} \mathrm{(deuteriochloroform):} \delta 36.9$ $\left(\mathrm{CH}_{2}\right), 90.5(\mathrm{C}), 109.1(\mathrm{C}), 122.7(\mathrm{CH}), 122.9(\mathrm{CH}), 125.9(2 \mathrm{CH}), 126.1(\mathrm{CH}), 127.9(\mathrm{CH})$, $128.1(2 \mathrm{CH}), 128.6(\mathrm{CH}), 129.2(\mathrm{CH}), 130.2(\mathrm{C}), 132.7(\mathrm{CH}), 137.9(\mathrm{C}), 139.4(\mathrm{C}), 149.7$ (C), 166.9 (CO); ms: (EI) m/z $400\left(\mathrm{M}^{+}\right)$.

Anal. Calcd. for $\mathrm{C}_{19} \mathrm{H}_{14} \mathrm{BrNO}_{2} \mathrm{~S}$ (400.29): C, 57.01; H, 3.53; N, 3.50. Found: C, 56.97; H, $3.40 ; \mathrm{N}, 3.39$.

2,3-Dihydro-3-phenyl-2-(2'-bromothien-3'-ylmethyl)-1H-isoindol-1-one (5c).

A solution of $4 \mathbf{c}(4.1 \mathrm{~g}, 10.24$ mmoles $)$ in a mixture of $10 \mathrm{ml}$ of trifluoroacetic acid and 20 $\mathrm{ml}$ of dry dichloromethane was stirred under an atmosphere of nitrogen and cooled in an ice bath. The solution was treated with $1.2 \mathrm{~g}$ (10.3 mmoles) of triethylsilane, added over a period of 5 minutes. After removal of this ice bath, the stirring was continued for 1 hour. The mixture was then poured into ice-water, made alkaline by the addition of ammonia and extracted with dichloromethane. The extracts were combined, dried and evaporated in vacuo. The oily residue was purified by flash chromatography on silica gel column eluting with dichloromethane/ligroin (9:1) $\left(\mathbf{R}_{\mathrm{f}} 0.82\right)$ to isolate $\omega$-carbinol lactam $\mathbf{5} \mathbf{c}$ as orange crystals in a yield of 94\%, ir (neat): $v 3070$ and $2958(\mathrm{CH}), 1697(\mathrm{C}=\mathrm{O}) \mathrm{cm}^{-1} ;{ }^{1} \mathrm{H} \mathrm{nmr}$ (deuteriochloroform): $\delta 3.97\left(\mathrm{~d}, 1 \mathrm{H}, \mathrm{J}=15.1 \mathrm{~Hz}, \mathrm{CH}_{2}-\mathrm{N}\right), 5.07\left(\mathrm{~d}, 1 \mathrm{H}, \mathrm{J}=15.1 \mathrm{~Hz}, \mathrm{CH}_{2}-\mathrm{N}\right), 5.25(\mathrm{~s}, 1 \mathrm{H}$,

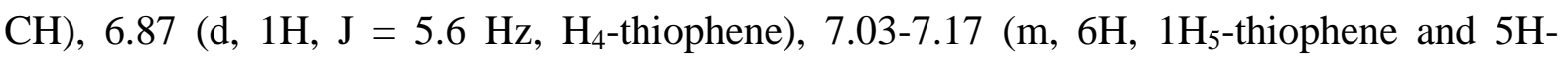
benzene), 7.27-7.33 (m, 2H, 2H-benzene), 7.8-7.9 (m, 2H, 2H-benzene); ${ }^{13} \mathrm{C} \mathrm{nmr}$ (deuteriochloroform): $\delta 33.2\left(\mathrm{CH}_{2}\right), 64.1(\mathrm{CH}), 111.2(\mathrm{C}), 123.1(\mathrm{CH}), 123.6(\mathrm{CH}), 126.2$ $(\mathrm{CH}), 127.6(2 \mathrm{CH}), 128.2(\mathrm{CH}), 128.6(2 \mathrm{CH}), 128.9(2 \mathrm{CH}), 130.9(\mathrm{C}), 131.9(\mathrm{CH}), 136.5$ (C), 136.7 (C), 146.4 (C), 168.6 (CO); ms: (EI) m/z $384\left(\mathrm{M}^{+}\right)$. 
Anal. Calcd. for $\mathrm{C}_{19} \mathrm{H}_{14} \mathrm{BrNOS}$ (384.29): C, 59.38; H, 3.67; N, 3.64. Found: C, 59.05; H, $3.63 ; \mathrm{N}, 3.62$.

General Procedure for Synthesis of Diarylimides $\mathbf{6 a , b , c}$.

To a stirred solution of $N$-alkylated imide 3a, 3b or 3c (20.6 mmoles) in dimethyl ether (40 $\mathrm{ml}$ ) under dry nitrogen was added slowly by portions tetrakis(triphenylphosphine)palladium (0.72 g, $0.6 \mathrm{mmole})$. After 30 minutes of reaction a solution of anhydrous potassium carbonate $(2.85 \mathrm{~g}, 20.6 \mathrm{mmoles})$ in water $(20 \mathrm{ml})$ and phenyl boronic acid $(3.02 \mathrm{~g}, 24.8$ mmoles) were added to the mixture which was then boiled under reflux. After 24 hours of reaction, the solvent was evaporated in vacuo and the aqueous solution was extracted with dichloromethane. Drying and evaporation of the extract gave a brown solid which was purified by flash chromatography on silica gel column (ethyl acetate/hexane).

1-(2'-Phenylthien-3'-ylmethyl)succinimide (6a).

This compound was isolated as a white solid by crystallization from diethyl ether in $98 \%$ yield, mp 98 $8^{\circ} \mathrm{R}_{\mathrm{f}} 0.55$ (ethyl acetate/diethyl ether, 3:1); ir: $v 2899(\mathrm{CH}), 1592(\mathrm{C}=\mathrm{O}) \mathrm{cm}^{-1} ;{ }^{1} \mathrm{H}$ nmr (deuteriochloroform): $\delta 2.60$ (s, 4H, 4H-succinimide), $4.70\left(\mathrm{~s}, 2 \mathrm{H}, \mathrm{CH}_{2}-\mathrm{N}\right), 6.95(\mathrm{~d}, 1 \mathrm{H}$, $\mathrm{J}=5.4 \mathrm{~Hz}, \mathrm{H}_{4}$--thiophene), $7.22\left(\mathrm{~d}, 1 \mathrm{H}, \mathrm{J}=5.4 \mathrm{~Hz}, \mathrm{H}_{5}\right.$,-thiophene), 7.35-7.45 (m, 3H, 3Hbenzene), 7.61-7.72 (m, 2H, 2H-benzene); ${ }^{13} \mathrm{C} \mathrm{nmr}$ (deuteriochloroform): $\delta 28.1\left(2 \mathrm{CH}_{2}\right), 36.5$ $\left(\mathrm{CH}_{2}\right), 124.6(\mathrm{CH}), 127.9(\mathrm{CH}), 128.3(\mathrm{CH}), 128.4(\mathrm{CH}), 128.7(\mathrm{CH}), 129.2(\mathrm{CH}), 129.7$ (CH), 131.2 (C), $133.4(\mathrm{C}), 140.9$ (C), 176.3 (2CO); ms: (EI) m/z $271\left(\mathrm{M}^{+}\right)$.

Anal. Calcd. for $\mathrm{C}_{15} \mathrm{H}_{13} \mathrm{NO}_{2} \mathrm{~S}$ (271.33): C, 66.40; H, 4.83; N, 5.16. Found: C, 66.23; H, $4.60 ; \mathrm{N}, 5.05$.

\section{3,3,4,4-Tetramethyl-1-(2'-phenylthien-3'-ylmethyl)succinimide (6b).}

This product was obtained in the same manner from $\mathbf{3 b}$ in $96 \%$ yield, $\mathrm{mp} 92^{\circ}$ (ethanol/diethyl ether); $\mathrm{R}_{\mathrm{f}} 0.82$ (ethyl acetate/diethyl ether, 4:1); ir: $v 2909(\mathrm{CH}), 1683(\mathrm{C}=\mathrm{O})$ $\mathrm{cm}^{-1} ;{ }^{1} \mathrm{H} \mathrm{nmr}$ (deuteriochloroform): $\delta 2.67\left(\mathrm{~s}, 12 \mathrm{H}, 4 \mathrm{CH}_{3}\right), 4.60\left(\mathrm{~s}, 2 \mathrm{H}, \mathrm{CH}_{2}-\mathrm{N}\right), 6.87(\mathrm{~d}, 1 \mathrm{H}$, $\mathrm{J}=5.4 \mathrm{~Hz}, \mathrm{H}_{4}$,-thiophene), $7.18\left(\mathrm{~d}, 1 \mathrm{H}, \mathrm{J}=5.4 \mathrm{~Hz}, \mathrm{H}_{5}\right.$,-thiophene), 7.22-7.37 (m, 3H, 3Hbenzene), 7.39-7.71 (m, 2H, 2H-benzene); ${ }^{13} \mathrm{C} \mathrm{nmr}$ (deuteriochloroform): $\delta 21.2\left(4 \mathrm{CH}_{3}\right), 36.2$ $\left(\mathrm{CH}_{2}\right), 46.7(2 \mathrm{C}), 124.6(\mathrm{CH}), 127.7(\mathrm{CH}), 127.8(\mathrm{CH}), 128.5(\mathrm{CH}), 129.6(\mathrm{CH}), 131.6(\mathrm{C})$, $131.9(\mathrm{CH}), 132.1(\mathrm{CH}), 133.4(\mathrm{C}), 140.9(\mathrm{C}), 182.5$ (2CO); ms: (EI) m/z $327\left(\mathrm{M}^{+}\right)$.

Anal. Calcd. for $\mathrm{C}_{19} \mathrm{H}_{21} \mathrm{NO}_{2} \mathrm{~S}$ (327.44): C, 69.69; H, 6.46; N, 4.28. Found: C, 69.51; H, $6.30 ; \mathrm{N}, 4.11$.

2-(2'-Phenylthien-3'-ylmethyl)phthalimide (6c). 
This product was obtained in the same manner from $3 \mathbf{c}$ in a yield of $95 \%, \mathrm{mp} 157^{\circ} ; \mathrm{R}_{\mathrm{f}} 0.70$ (dichloromethane/diethyl ether, 4:1); ir: $v 3085$ and $2909(\mathrm{CH}), 1703(\mathrm{C}=\mathrm{O}) \mathrm{cm}^{-1} ;{ }^{1} \mathrm{H} \mathrm{nmr}$ (deuteriochloroform): $\delta 4.85$ (s, 2H, $\left.\mathrm{CH}_{2}-\mathrm{N}\right), 7.00$ (d, 1H, J = 5.4 Hz, $\mathrm{H}_{4}$,-thiophene), 7.16 (d, $1 \mathrm{H}, \mathrm{J}=5.4 \mathrm{~Hz}, \mathrm{H}_{5}$-thiophene), 7.25-7.50 (m, 3H, 3H-benzene), 7.52-7.61 (m, 2H, 2Hbenzene), 7.63-7.70 (m, 2H, 2H-phthalimide), 7.71-7.80 (m, 2H, 2H-phthalimide); ms: (EI) $\mathrm{m} / \mathrm{z} 319\left(\mathrm{M}^{+}\right)$.

Anal. Calcd. for $\mathrm{C}_{19} \mathrm{H}_{13} \mathrm{NO}_{2} \mathrm{~S}$ (319.37): C, 71.45; H, 4.10; N, 4.39. Found: C, 71.31; H, $4.00 ;$ N.4.22.

General Procedure for Reduction of Imides $\mathbf{6 a , b , c . ~}$

To a stirred solution of $N$-alkylated imide $\mathbf{6 a}, \mathbf{6 b}$ or $\mathbf{6 c}$ (12.6 mmoles) in dry methanol (15 $\mathrm{ml})$ was added slowly in portions sodium borohydride $(2.83 \mathrm{~g}, 75 \mathrm{mmoles})$ at $-5-0^{\circ}$ over a period of 10 minutes. While the temperature was kept at $-5-0^{\circ}, 2 \mathrm{~N}$ hydrochloric acid solution in dry ethanol ( 3 drops) was added to the reaction mixture at regular intervals of 10 minutes during 3 hours. The excess of sodium borohydride was destroyed by adding cold water (10 $\mathrm{ml}$ ) then a solution of $10 \%$ hydrochloric acid in dry ethanol at $0-5^{\circ}$ to $p \mathrm{H} 3$. After removal of the solvent, the residue was diluted with water $(45 \mathrm{ml})$ and extracted with dichloromethane. The organic layers were washed with saturated brine, dried over sodium sulfate and evaporated in vacuo. The oily residue after trituration with diethyl ether was recrystallized from ethanol to give $\omega$-carbinol lactams $\mathbf{7 a}, \mathbf{7 b}$ or $\mathbf{7 c}$.

1-(2'-Phenylthien-3'-ylmethyl)succinamidal (7a).

This product was obtained as yellow crystals in $85 \%$ yield, mp $148^{\circ} ; R_{\mathrm{f}} 0.75$ (dichloromethane/acetone, 9:1); ir: $v 3345$ (br, O-H), $1662(\mathrm{C}=\mathrm{O}) \mathrm{cm}^{-1} ;{ }^{1} \mathrm{H} \mathrm{nmr}$ (deuteriochloroform): $\delta$ 0.75-0.95 (m, 1H, 1H-pyrrolidinone), 1.13-2.22 (m, 1H, 1Hpyrrolidinone), 1.62-1.93 (m, 1H, 1H-pyrrolidinone), 1.97-2.21 (m, 1H, 1H-pyrrolidinone), $3.78(\mathrm{~d}, 1 \mathrm{H}, \mathrm{J}=15 \mathrm{~Hz}, \mathrm{CH}-\mathrm{N}), 3.76-3.81(\mathrm{~m}, 1 \mathrm{H}, 1 \mathrm{H}-$ pyrrolidinone), 4.91 (d, 1H, J = $15 \mathrm{~Hz}$, $\left.\mathrm{CH}_{2}-\mathrm{N}\right), 6.82\left(\mathrm{~d}, 1 \mathrm{H}, \mathrm{J}=5.4 \mathrm{~Hz}, \mathrm{H}_{4}\right.$-thiophene), 7.07 (d, 1H, J = 5.4 Hz, $\mathrm{H}_{5}$-'thiophene), 7.09 $(\mathrm{d}, \quad 1 \mathrm{H}, \quad \mathrm{J}=7.8 \mathrm{~Hz}, \quad 1 \mathrm{H}$-benzene $), \quad 7.30-7.35 \quad(\mathrm{~m}, 4 \mathrm{H}, 4 \mathrm{H}$-benzene $) ;{ }^{13} \mathrm{C} \mathrm{nmr}$ (deuteriochloroform): $\delta 25.5\left(\mathrm{CH}_{2}\right), 28.4\left(\mathrm{CH}_{2}\right), 36.3\left(\mathrm{CH}_{2}\right), 88.4(\mathrm{CH}), 125.3(\mathrm{CH}), 128.1$ $(\mathrm{CH}), 128.4(\mathrm{CH}), 128.8(2 \mathrm{CH}), 129.6(2 \mathrm{CH}), 132.1(\mathrm{C}), 133.1(\mathrm{C}), 140.9(\mathrm{C}), 174.2(\mathrm{CO})$; ms: (EI) m/z $273\left(\mathrm{M}^{+}\right)$.

Anal. Calcd. for $\mathrm{C}_{15} \mathrm{H}_{15} \mathrm{NO}_{2} \mathrm{~S}$ (273.35): C, 65.91; H, 5.53; N, 5.12. Found: C, 65.81; H, $5.35 ; \mathrm{N}, 5.09$.

3,3,4,4-Tetramethyl-1-(2'-phenylthien-3'-ylmethyl)succinamidal (7b). 
This compound was isolated after 24 hours of reaction at room temperature from $\mathbf{6 b}$ as colorless solid in $96 \%$ yield, mp $154^{\circ} ; \mathrm{R}_{\mathrm{f}} 0.66$ (dichloromethane/hexane, 9:1); ir: $v 3224$ (br, $\mathrm{O}-\mathrm{H}), 1651(\mathrm{C}=\mathrm{O}) \mathrm{cm}^{-1} ;{ }^{1} \mathrm{H} \mathrm{nmr}$ (deuteriochloroform): $\delta 0.77$ (s, 3H, $\left.\mathrm{CH}_{3}\right), 0.87\left(\mathrm{~s}, 3 \mathrm{H}, \mathrm{CH}_{3}\right)$, 0.92 (s, 3H, $\left.\mathrm{CH}_{3}\right), 1.11\left(\mathrm{~s}, 3 \mathrm{H}, \mathrm{CH}_{3}\right), 4.31\left(\mathrm{~d}, 1 \mathrm{H}, \mathrm{J}=15 \mathrm{~Hz}, \mathrm{CH}_{2}-\mathrm{N}\right), 4.33-4.36(\mathrm{~m}, 1 \mathrm{H}, 1 \mathrm{H}-$ pyrrolidinone), $4.82\left(\mathrm{~d}, 1 \mathrm{H}, \mathrm{J}=15 \mathrm{~Hz}, \mathrm{CH}_{2}-\mathrm{N}\right), 6.96\left(\mathrm{~d}, 1 \mathrm{H}, \mathrm{J}=5.2 \mathrm{~Hz}, \mathrm{H}_{4}\right.$--thiophene), 7.21 (d, 1H, J = 5.2 Hz, H5,-thiophene), 7.35-7.42 (m, 4H, 4H-benzene), 7.56-7.62 (m, 1H, 1Hbenzene); ${ }^{13} \mathrm{C} \mathrm{nmr}$ (deuteriochloroform): $\delta 17.2\left(\mathrm{CH}_{3}\right), 19.3\left(\mathrm{CH}_{3}\right), 23.2\left(\mathrm{CH}_{3}\right), 23.6\left(\mathrm{CH}_{3}\right)$, $37.3\left(\mathrm{CH}_{2}\right), 42.3(\mathrm{C}), 45.8(\mathrm{C}), 88.2(\mathrm{CH}), 124.7(\mathrm{CH}), 128.0(\mathrm{CH}), 128.8(2 \mathrm{CH}), 129.1(\mathrm{CH})$, $129.4(2 \mathrm{CH}), 132.7$ (C), 133.5 (C), 140.8 (C), 178.5 (CO); ms: (EI) m/z $329\left(\mathrm{M}^{+}\right)$.

Anal. Calcd. for $\mathrm{C}_{19} \mathrm{H}_{23} \mathrm{NO}_{2} \mathrm{~S}$ (329.45): C, 69.27; H, 7.04; N, 4.25. Found: C, 69.11; H, $6.95 ; \mathrm{N}, 4.15$.

2,3-Dihydro-3-hydroxy-2-(2'-phenylthien-3'-ylmethyl)-1H-isoindol-1-one (7c).

This compound was isolated as a white solid in $96 \%$ yield, $\mathrm{mp} 155^{\circ} ; \mathrm{R}_{\mathrm{f}} 0.66$ (dichloromethane/hexane, 9:1); ir: $v 3313$ (br, OH), $1658 \quad(\mathrm{C}=\mathrm{O}) \quad \mathrm{cm}^{-1} ;{ }^{1} \mathrm{H} \mathrm{nmr}$ (deuteriochloroform): $\delta 2.73(\mathrm{~d}, 1 \mathrm{H}, \mathrm{J}=10.8 \mathrm{~Hz}, \mathrm{OH}), 4.42\left(\mathrm{~d}, 1 \mathrm{H}, \mathrm{J}=15.1 \mathrm{~Hz}, \mathrm{CH}_{2}-\mathrm{N}\right)$, $4.88\left(\mathrm{~d}, 1 \mathrm{H}, \mathrm{J}=15.1 \mathrm{~Hz}, \mathrm{CH}_{2}-\mathrm{N}\right), 5.55(\mathrm{~d}, 1 \mathrm{H}, \mathrm{J}=10.8 \mathrm{~Hz}, 1 \mathrm{H}$-isoindole), 6.95 (d, 1H, J = 5.1 $\mathrm{Hz}, \mathrm{H}_{4}$-thiophene), 7.19 (d, 1H, J = 5.1 Hz, H${ }_{5}$-thiophene), 7.30-7.55 (m, 8H, 8H-benzene), 7.56 (d, 1H, J = $7 \mathrm{~Hz}, 1 \mathrm{H}$-benzene); ms: (EI) m/z $321\left(\mathrm{M}^{+}\right)$.

Anal. Calcd. for $\mathrm{C}_{19} \mathrm{H}_{15} \mathrm{NO}_{2} \mathrm{~S}$ (321.39): C, 71.01; H, 4.70; N, 4.36. Found: C, 70.92; H, $4.55 ; \mathrm{N}, 4.29$.

General Procedure for the Synthesis of Pyrrolo(or isoindolo)thienobenzazepines $\mathbf{8 a}, \mathbf{8 b}$ and $8 c$.

To a stirred solution of hydroxylactam $\mathbf{7 a}, 7 \mathbf{b}$ or $\mathbf{7 c}(2 \mathrm{mmoles})$ in dichloromethane $(50 \mathrm{ml})$ was added trifluoroacetic acid $(6 \mathrm{ml})$. After 24 hours of reaction at room temperature, the reaction mixture was diluted with water $(50 \mathrm{ml})$ and neutralized with an aqueous $10 \%$ sodium hydroxide solution. The organic layer was separated, washed with water $(50 \mathrm{ml})$, dried over magnesium sulfate and concentrated in vacuo to give after flash chromatography (silica gel; dichloromethane/hexane, 9:1) and recrystallization from suitable solvent the expected tetracyclic products $\mathbf{8 a - c .}$

2,3,3a,11-Tetrahydropyrrolo[2,1-a]thieno[3,2-d][2]benzazepin-1-one (8a).

This product was obtained as a white solid in $80 \%$ yield, $\mathrm{mp} 128^{\circ}$ (ethanol); $\mathrm{R}_{\mathrm{f}} 0.74$ (chloroform/ligroin, 8:2); ir: $v 2894(\mathrm{CH}), 1673(\mathrm{C}=\mathrm{O}) \mathrm{cm}^{-1} ;{ }^{1} \mathrm{H} \mathrm{nmr}$ (deuteriochloroform): $\delta$ 
2.24-2.59 (m, 4H, 4H-pyrrolidinone), $3.74\left(\mathrm{~d}, 1 \mathrm{H}, \mathrm{J}=14.5 \mathrm{~Hz}, \mathrm{CH}_{2}-\mathrm{N}\right), 4.51(\mathrm{dd}, 1 \mathrm{H}, \mathrm{J}=8$ $\mathrm{Hz}$ and $\mathrm{J}=13.6 \mathrm{~Hz}, 1 \mathrm{H}$-pyrrolidinone), $4.55\left(\mathrm{~d}, 1 \mathrm{H}, \mathrm{J}=14.5 \mathrm{~Hz}, \mathrm{CH}_{2}-\mathrm{N}\right), 7.03(\mathrm{~d}, 1 \mathrm{H}, \mathrm{J}=5.2$ $\mathrm{Hz}, \mathrm{H}_{4}$,-thiophene), 7.21-7.23 (m, 1H, 1H-benzene), 7.28 (d, 1H, J = 5.2 Hz, $\mathrm{H}_{5}$--thiophene), 7.35-7.49 (m, 3H, 3H-benzene); ${ }^{13} \mathrm{C} \mathrm{nmr}$ (deuteriochloroform): $\delta 22.6\left(\mathrm{CH}_{2}\right), 31.5\left(\mathrm{CH}_{2}\right)$, $39.7\left(\mathrm{CH}_{2}\right), 58.5(\mathrm{CH}), 125.3(\mathrm{CH}), 125.6(\mathrm{CH}), 128.1(\mathrm{CH}), 128.5(\mathrm{CH}), 128.9(\mathrm{CH}), 129.1$ (CH), 133.9 (C), 134.7 (C), 135.1 (C), 139.8 (C), 173.1 (CO); ms: (EI) m/z $255\left(\mathrm{M}^{+}\right)$.

Anal. Calcd. for $\mathrm{C}_{15} \mathrm{H}_{13} \mathrm{NOS}$ (255.33): C, 70.59; H, 5.13; N, 5.49. Found: C, 70.34; H, 5.05; N, 5.29.

2,3,3a,11-Tetrahydro-2,2,3,3-tetramethylpyrrolo[2,1-a]thieno[3,2- $d][2]$ benzazepin-1-one $(\mathbf{8 b})$.

This product was obtained similarly from $7 \mathbf{b}$ in a yield of $85 \%, \mathrm{mp} 130^{\circ}$ (ethanol); $\mathrm{R}_{\mathrm{f}} 0.85$ (ethyl acetate/hexane, 9:1); ir: $\vee 2978(\mathrm{CH}), 1680(\mathrm{C}=\mathrm{O}) \mathrm{cm}^{-1} ;{ }^{1} \mathrm{H} \mathrm{nmr}$ (deuteriochloroform): $\delta 0.19\left(\mathrm{~s}, 3 \mathrm{H}, \mathrm{CH}_{3}\right), 0.88\left(\mathrm{~s}, 3 \mathrm{H}, \mathrm{CH}_{3}\right), 0.91\left(\mathrm{~s}, 3 \mathrm{H}, \mathrm{CH}_{3}\right), 1.10\left(\mathrm{~s}, 3 \mathrm{H}, \mathrm{CH}_{3}\right), 3.73(\mathrm{~d}, 1 \mathrm{H}, \mathrm{J}=$ 14.2 Hz, $\mathrm{CH}_{2}-\mathrm{N}$ ), 4.96 (s, 1H, 1H-pyrrolidinone), 3.73 (d, 1H, J = 14.2 Hz, $\mathrm{CH}_{2}-\mathrm{N}$ ), 7.03 (d, $1 \mathrm{H}, \mathrm{J}=5.2 \mathrm{~Hz}, \mathrm{H}_{4}$--thiophene), 7.13 (d, $1 \mathrm{H}, \mathrm{J}=5.2 \mathrm{~Hz}, \mathrm{H}_{5}$-thiophene), 7.28-7.33 (m, 2H, 2H-benzene), 7.50-7.55 (m, 2H, 2H-benzene); ${ }^{13} \mathrm{C} \mathrm{nmr} \mathrm{(deuteriochloroform):} \delta 18.1\left(\mathrm{CH}_{3}\right)$, $18.8\left(\mathrm{CH}_{3}\right), 18.9\left(\mathrm{CH}_{3}\right), 20.6\left(\mathrm{CH}_{3}\right), 38.5\left(\mathrm{CH}_{2}\right), 47.1(\mathrm{C}), 47.4(\mathrm{C}), 72.4(\mathrm{CH}), 123.9(\mathrm{CH})$, $126.6(\mathrm{CH}), 128.2(\mathrm{CH}), 128.5(\mathrm{CH}), 130.4(\mathrm{CH}), 130.8(\mathrm{CH}), 133.9(\mathrm{C}), 134.2(\mathrm{C}), 138.2$ (C), 139.7 (C), 177.9 (CO); ms: (EI) m/z $311\left(\mathrm{M}^{+}\right)$.

Anal. Calcd. for $\mathrm{C}_{19} \mathrm{H}_{21}$ NOS (311.44): C, 73.27; H, 6.80; N, 4.50. Found: C, 73.18; H, 6.59; $\mathrm{N}, 4.22$.

4,10b-Dihydroisoindolo[1,2-a]thieno[3,2- $d][2]$ benzazepin-6-one (8c).

This product was obtained similarly from $7 \mathrm{c}$ in a yield of $88 \%, \mathrm{mp} 241^{\circ}$ (ethanol); $\mathrm{R}_{\mathrm{f}} 0.76$ (dichloromethane/hexane, 9:1); ir: $\quad$ v $2985 \quad(\mathrm{CH}), \quad 1684 \quad(\mathrm{C}=\mathrm{O}) \quad \mathrm{cm}^{-1} ; \quad{ }^{1} \mathrm{H} \quad \mathrm{nmr}$ (deuteriochloroform): $\delta 4.07\left(\mathrm{~d}, 1 \mathrm{H}, \mathrm{J}=15 \mathrm{~Hz}, \mathrm{CH}_{2}-\mathrm{N}\right), 5.20\left(\mathrm{~d}, 1 \mathrm{H}, \mathrm{J}=15 \mathrm{~Hz}, \mathrm{CH}_{2}-\mathrm{N}\right), 5.60$ (s, 1H, CH), 6.99-7.77 (m, 7H, 7H-benzene), 7.13 (d, 1H, J = 5 Hz, H ,-thiophene), 7.38 (d, $1 \mathrm{H}, \mathrm{J}=5 \mathrm{~Hz}, \mathrm{H}_{5}$-thiophene), $7.95\left(\mathrm{~d}, 1 \mathrm{H}, \mathrm{J}=6 \mathrm{~Hz}, 1 \mathrm{H}\right.$-benzene); ${ }^{13} \mathrm{C} \mathrm{nmr}$ (deuteriochloroform): $\delta 40.4\left(\mathrm{CH}_{2}\right), 61.4(\mathrm{CH}), 123.9(\mathrm{CH}), 124.5(\mathrm{CH}), 125.6(\mathrm{CH}), 126.7(\mathrm{CH})$, $128.2(\mathrm{CH}), 128.6(\mathrm{CH}), 128.9(\mathrm{C}), 129(\mathrm{CH}), 129.3(\mathrm{CH}), 131(\mathrm{CH}), 134(\mathrm{C}), 134.2(\mathrm{C})$, 134.5 (C), 135 (C), 139.7 (C), 141.9 (CH), 166.4 (CO); ms: (EI) m/z 303 (M+).

Anal. Calcd. for $\mathrm{C}_{19} \mathrm{H}_{13} \mathrm{NOS}$ (303.38): C, 75.22; H, 4.32; N, 4.62. Found: C, 75.09; H, 4.25; $\mathrm{N}, 4.55$. 


\section{REFERENCES AND NOTES}

[1] P. Pigeon and B. Decroix, J. Heterocyclic Chem., 33, 129 (1996).

[2] A. Mamouni, A. Daïch and B. Decroix, J. Heterocyclic Chem., 33, 1251 (1996).

[3] H. O. Bernhard and V. Snieckus, Tetrahedron Letters. 4867 (1971); E. Valencia, I. Weiss, S. Firdous, A. J. Freyer, M. Shamma, A. Urzùa and V. Fajardo, Tetrahedron, 40, 3957 (1984); E. Valencia, V. Fajardo, A. J. Freyer and M. Shamma, Tetrahedron Letters, 26, 993 (1985); P. H. Mazzocchi, C. R. King and H. L. Aammon, Tetrahedron Letters, 28, 2473 (1987); S. V. Kessar, T. Singh and R. Vohra, Tetrahedron Letters, 28, 5323 (1987); R. Alonso, L. Castedo and D. Dominguez, Tetrahedron Letters, 26, 2925 (1985); C. Lamas, C. Saa, L. Castedo and D. Dominguez, Tetrahedron Letters, 33, 5653 (1992); G. Rodriguez, M. M. Cid, C. Saà, L. Castedo and D. Dominguez, J. Org. Chem., 61, 2780 (1996).

[4] S. M. Weinreb and M. F. Semmelhack, Acc. Chem. Res., 8, 158 (1975).

[5] W. J. Houlihan and J. Nadelson. U.S. Patent 3892752 (1975); Chem. Abstr., 83, 178856k (1975).

[6] W. J. Van der Burg, I. L. Bonta, J. Delobelle, C. Ramon and B. Vargaftig, J. Med. Chem., 13, 35 (1970), W. F. Kafoe, J. J. De Ridder and B. F. Leonard, Biochem. Pharm., 25, 2455 (1976).

[7] B. E. Maryanoff, D. E. McComsey, J. E. Gardocki, R. P. Shank, M. J. Costanzo, S. O. Nortey, C. R. Schneider and P. Seller, J. Med. Chem., 30, 1433 (1987).

[8] I. Hall. A. R. K. Murthy and S. D. Wyrick, J. Pharm. Sci., 75, 622 (1986).

[9] G. Steiner, A. Franke, E. Haedicke, D. Lenke, H. Teschendorf, H. Hofmann, H. Kreiskott and W. Worstmann, J. Med. Chem., 29, 1877 (1986).

[10] H. Wunderlich, A. Strak, E. Carstens, D. Lohmann, A. N, Gritsenko and A. P. Skoldinov, Pharmazie, 40, 827 (1985).

[11] P. Pigeon and B. Decroix, Tetrahedron Letters, 37, 7707 (1996).

[12] H. Ishibashi, H. Kawanami, H. Iriyama and M. Ikeda, Tetrahedron Letters, 36, 6733 (1995).

[13] M. Ikeda, S. Akamatsu, Y. Kugo and T. Sato, Heterocycles, 42, 155 (1996).

[14] D. L. Comins, S. P. Joseph and Y. Zhang, Tetrahedron Letters, 37, 793 (1996). 
[15] B. K. Banik, G. V. Subbaraju, M. S. Manhas and A. K. Bose. Tetrahedron Letters, 37, 1363 (1996).

[16] W. Speckamp and H. Hiemstra, Tetrahedron, 41, 4367 (1985).

[17] P Pigeon and B. Decroix, Tetrahedron Letters, 38, 1041 (1997).

[18] J. E. Baldwin, J. Chem. Soc., Chem. Commun., 734 (1976).

[19] A. F. Bickel and W. A. Waters, Rec. Trav. Chim. Pays Bas, 69, 1490 (1950).

[20] F. F. Blicke and D. G. Sheets. J. Am. Chem. Soc., 70, 3768 (1948).

[21] J. P. Gesson, J. C. Jacquesy and D. Rambaud, Bull. Soc. Chim. France, 129, 227 (1992).

[22] J. B. P. A. Wijnberg, H. E. Schoemaker and W. N. Speckamp, Tetrahedron, 34, 179 (1978).

[23] J. C. Hubert, J. B. P. A. Wijnberg and W. N. Speckamp, Tetrahedron, 31, $1437(1975)$. 